\title{
A SURVEY ON IMAGE BASED EYE-BLINK ASSISTIVE SYSTEM FOR PARALYZED PATIENTS
}

\author{
Mrs.Vijayalaxmi Mekali ${ }^{1}$, Vidhya Dhari L ${ }^{2}$, Sandra Vaanija ${ }^{3}$, Supriya $C^{4}$ \\ ${ }^{1}$ Associate Professor, Dept. of Computer Science and Engineering \\ 2,3,4 Department of Computer Science and Engineering \\ 1,2,3,4 K S Institute of Technology, Bangalore, India
}

\begin{abstract}
The main aim of this paper is to remove difficulties faced by completely paralyzed patients suffering from Motor Neuron Disease MND) and Locked-in Syndrome (LIS). Paralyzed patients cannot communicate as they suffer from speech disorder, the only part that remains unaffected is eyes and communication is possible only through their eye movements. The proposed system is based on a Video-Oculography (VOG) technique which is efficient when compared to other existing techniques. In this system methods like face detection, eye blink detection and image processing are employed to communicate the needs of patients to the concerned person through a text and an audio message.
\end{abstract}

Keywords - Motor Neuron Disease, Locked-in Syndrome, Speech Disorder, Video-Oculography, Face Detection, Eye Blink Detection, Image Processing.

\section{INTRODUCTION}

Motor Neuron Disease (MND) and Locked-in Syndrome(LIS) are incurable medical conditions where the patient is completely paralyzed. It also leads to inability to communicate through speech. Because of this, performing voluntary actions is not possible and it becomes very difficult for the patients to express their needs. A caretaker has to assist the patient 24/7. In most of the cases the caretaker might not understand what the patient is trying to convey.

Many systems have been developed to address this issue. Currently the technology used is Brain wave controlling and Electro-oculography. Both the methods are expensive and also uncomfortable to wear. In Electro-oculography the electrodes are pierced through the skin which is quite painful.

The proposed method uses Video-Oculography technique which is less expensive, comfortable and not painful. In the proposed system, firstly the patient's face is detected and extraction of eye region is done using Face Detection algorithm. Then, eye-blink detection is done using Eye-Blink algorithm.

Paralyzed patients should choose an image from the set of stored images being scrolled by blinking their eyes voluntarily as per their requirement. The chosen image is communicated to the concerned person as the patient's requirement through an audio and a text message on their phone.

We use OpenCV-Python for face and eye detection. Using such image processing and machine learning techniques, the patient's intent or needs can be conveyed more effectively and efficiently.

\section{LITERATURE SURVEY}

Tambe Samreen Mohammed et al. (2019) proposed a review on different methods or techniques used for solving MND patient problems especially speech disorder problem. The authors specially talk about the advantages of using Videooculography technique over the existing methods.

Hashim N A et al. (2018) proposed a technique that can assist paralyzed patients by tracking person's eye and counting the blinks, and employs this count to control various appliances and play pre-recorded audio messages.

Kingshuk Mukherjee et al. (2015) proposed an extremely low priced device that reads and converts eye-blinks from the patient to a universally accepted communication code-The Morse code. Readings are taken from IR sensor and are converted to a communication code.

Guruprasad M Bhat et al. (2017) proposed an assistive wheel chair system for paralyzed patients using eye gaze methodology. The wheelchair moves based on the commands received from the system through eye gaze movements. This 


\section{International Journal of Engineering Applied Sciences and Technology, 2019 Vol. 4, Issue 8, ISSN No. 2455-2143, Pages 362-364 \\ Published Online December 2019 in IJEAST (http://www.ijeast.com)}

system is built using hardware components like Raspberry Pi and software includes algorithms of Artificial intelligence and machine learning.

Sabu George et al. (2017) proposed a psychological experiment for deception detection where the blink count and duration was analysed while answering the control questions. It was analysed that the blink duration is more while lying.

Atish Udayashankar et al. (2012) proposed an algorithm for face detection and eye tracking. The authors describe the various phases in implementing the system where the phases involve detecting eye position and then counting number of eye blink and converting into commands to control home appliances.

Milan Pandey et al. (2018) proposed methodology used for developing a system to assist MND patients to communicate or express their needs in an efficient way using eye tracking methodology specifically locating the position of eye pupil.

Sudhir Rao Rupanagudi et al. (2014) proposed a new proposition, where simple eye blinks could be used by the paralyzed individuals in order to communicate. These blinks further on could be converted to Morse code to transmit messages, where in each blink represents a dot or a dash using Novel algorithm.

Veena $\mathrm{N}$ et al. (2019) proposed a system which permits physically handicapped patients to convert their intent to text using their eye blinks. A computer system which is vision based is used for recognition of voluntary eye-blinks, along with the application of HCI for people with physical incapacities. The identified eye blink is considered as the response and the selected character is converted to text.

V. Kovendan (2019) proposed a system which makes paralyzed paitents simple to use their room environment by their own without others help. Based on eye movement control, smart appliances can be controlled when the patient is alone. The author proposed a novel EOG-based automated switch design, in which a visual trigger mechanism is introduced to guide the users' blinks and to assist in detecting eye blinks based on the GUI, which includes a switch button that flashes per every time interval.

Kishore Kumar G et al. (2019) proposed that an Eye blink detection is a very challenging problem due to the movement of the eyes and the variation of light. The authors proposed a method which provides a better improvement for eye detection and blinking. Artificial light is used to improve the accuracy of detection as well as the blinking for a distance equal to 13 inches. Using the hardware each frame takes an average of 60$70 \mathrm{~ms}$ for execution which is very efficient for real time application.
Aree A. Mohammed et al. (2014) proposed a real time method based on some video and image processing algorithms for eye blink detection. The motivation of this research is the need of disabling who cannot control the calls with human mobile interaction directly without the need of hands. Eye blinking detection based on eyelids state (close or open) is used for controlling android mobile phones.

\section{CONCLUSION}

The proposed system enables people suffering from Motor Neuron Disease(MND) and Locked-in Syndrome(LIS) to communicate in an effective and efficient way. This system is intended to assist the paralyzed and physically challenged to lead a normal life by controlling things and communicating their needs with less effort. It can be used by normal humans as well. The proposed method is efficient compared to existing ones because in the existing methods either electrodes are pierced or eye blink count is analysed. It is quite hard for the patient to remember the blink count associated with each of the image. Hence the proposed method solves this issue by considering the duration of eye blink wherein the patient's normal eye blink and voluntary eye blink for choosing a image(requirement) is differentiated with a pre-set threshold value.

\section{REFERENCES}

[1] Tambe Samreen Mohammed; Rajeshwari P (March 2019). "Review on Smart Eye Blink Solution for MND Patients Using Python" Volume 4, Issue 3 IJSDR.

[2] Hashim N A; Jithin K Manikandan; Sreerag Sajeev; Jisha P Abraham (May 2018). "Assitive Technology for People with Complete Paralysis”. Volume 5, Issue 5 IRJET.

[3] Kingshuk Mukherjee; Debdatta Chatterjee(2015). "Augmentative and Alternative Communication Device Based on Eye-Blink Detection and Conversion to Morse-Code to Aid Paralyzed Individuals".IEEE International Conference on Communication, Information \& Computing Technology (ICCICT).

[4] Guruprasad M Bhat; Abhilash A; Shivakumar M; Vinay B; Santosh D(May 2017). "Eye Gaze Recognition System To Assist Paralyzed Patients" Volume 1, Issue 2 PiCES International Journal.

[5] Sabu George; Manohara Pai M.M; Radhika M Pai; Samir Kumar Praharaj (2017). "Eye Blink Count And Eye Blink Duration Analysis For Deception Detection". IEEE International Conference on Advances in Computing, Communications and Informatics (ICACCI). 
[6] Atish Udayashankar; Amit R Kowshik; Chandramouli S; H S Prashanth (2012). "Assitance for the Paralysed Using Eye Blink Detection".IEEE $4^{\text {th }}$ International Conference on Digital Home.

[7] Milan Pandey; Kushal Chaudhari; Rajnish Kumar; Anoop Shinde; Divyanshu Totla; Prof. N.D. Mali (2018). "Assistance for Paralysed Patients Using Eye Motion Detection" IEEE International Conference.

[8] Sudhir Rao Rupanagudi; Vikas N S; Vivek C Bharadwaj; Manju; Dhruva N; Sowmya K S (2014). "Novel Methodology for Blink Recognition using Video Oculography for Communicating" IEEE International Conference.

[9] Veena N; Anitha N (July 2019). "Converting Intent of Physically Impaired into Text using Eye Blink Detection" Volume 8 Issue 2 IJRTE.

[10] V Kovendan (31 May 2019). "Eye Blinking Detection Based Emergency Alert and Automated Smart Environment for Patients with Severe Disorder" Volume 7 Issue 5 IJCSE.

[11] Kishore Kumar G; Kemparaju N; Parineetha S; Praveen J S; Tanuja P; Akalpitha L Kulkarni (May 2019). "Eye Waver Technology Based Assistive System For Disabled" IJLTEMAS.

[12] Aree A Mohammed; Shereen A Anwer (2014). "Efficient Eye Blink Detection Method For Disabled-Helping Domain" IJACSA. 